\title{
KERNFORSCHUNGSZENTRUM
}

\author{
KARLSR U HE
}

Institut für Kernverfahrenstechnik

Direkte Umwandlung von Kernenergie

in chemische Energie

U. Schindewolf

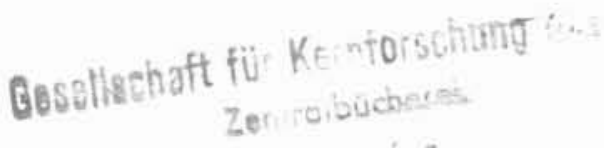

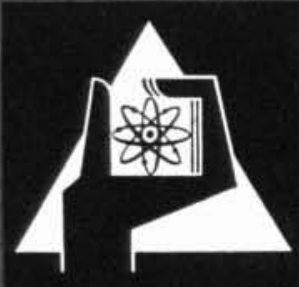




\title{
SONDERDRUCK aus der Zeitschrift \\ CHEMIE-INGENIEUR-TECHNIK
}

Zeitschrift für technische Chemie, Verfahrenstechnik und Apparatewesen

Verlag Chemie GmbH · Weinheim/Bergstr.

\section{Direkte Umwandlung von Kernenergie in chemische Energie}

\author{
Von Dr. U. Schindewolf
}

Kernforschungszentrum Karlsruhe, Institut für Kernverfahrenstechnik der T. H. Karisruhe

Casellschaft für Kernforschung m.h.t.

Zentralbücherei

1. Juli 1964
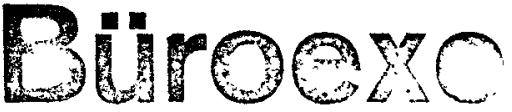

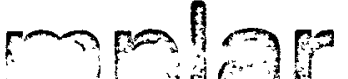

Nr.

Gesellschaft für Karnforse l.ng n.b.H. Karlsruhe 


\title{
Direkte Umwandlung von Kernenergie in chemische Energie
}

\author{
VON DR. U. SCHINDEWOLF \\ KERNFORSCHUNGSZENTRUM KARLSRUHE, \\ INSTITUT FOR KERNVERFAHRENSTECHNIK DER T.H. KARLSRUHE
}

\begin{abstract}
Durch strahlenchemische Prozesse lassen sich endotherme chemische Reaktionen erzwingen, in denen die Strahlenenergie teilweise in chemische Energie umgewandelt wird. Dadurch lassen sich aus einfachen Ausgangsstoffen wertvollere Produkte gewinnen. Als wirtsctuftliche Strahlenquellen kommen hierfür nur Kernreaktoren (Chemie-Kernreaktoren) in Frage. Da jedoch nur wenige Prozente der gesamten Reaktorenergie als chemische Energie gebunden werden, muB auch die verbleibende Reaktorenergie genützt werden. Ein Chemje-Kernreaktor wird nur dann wirtschaftich arbeiten, wenn der Wert des erzeugten chemischen Produktes mit dem Wert der erzeugien Energie mindestens vergleichbar ist.
\end{abstract}

Die unvermeidlichen radioaktiven Verunreinigungen der erzeugten chemischen Verbindungen dürften der großtechnischen Anwendung von Chemie-Kernreaktoren enge Grenzen ziehen.

Nach einer Betrachtung der Grundlagen chemonuclearer Prozesse werden die technischen Moglichkeiten und die wirtschaitlichen Aussichten der Synthese von Glykol aus Methanol und von Stickoxyden aus Luft diskutiert.

In allen hochindustrialisierten Ländern ist der Wert der chemischen Produkte, die durch endotherme, also Energie verbrauchende Reaktionen gewonnen werden, fast zehnmal höher als der Wert der erzeugten elektrischen Energie. Neben der Umwandlung von Kernenergie in elektrische Energie sind deshalb auch Verfahren von Interesse, die die direkte Umwandlung von Kernenergie in chemische Energie ermöglichen. Ein solches Verfahren setzt voraus, daß man die bei Kernumwandlungen frei werdenden energiereichen Kernstrahlen, also die $a$-, $\beta$ - und $\gamma$-Strahlen, Röntgenstrahlen, Rückstoßkerne der Kernspaltung und schnellen Neutronen, direkt auf chemische Systeme einwirken lassen kann und dadurch chemische Reaktionen erzwingt, die von allein aus energetischen Gründen nicht ablaufen können ${ }^{+}$.

Strahlenchemische Reaktionen, die nur unter ständiger Aufnahme von Energie verlaufen (in der Literatur auch chemonucleare Reaktionen genannt), wie $z$. B. die Radiolyse des Wassers

$2 \mathrm{H}_{2} \mathrm{O} \longrightarrow \mathrm{H}_{2}+\mathrm{H}_{2} \mathrm{O}_{2}$

sind seit der Entdeckung der Radioaktivität bekannt'); es wird heute sogar angenommen, daß die erste Synthese komplizierterer organischer Verbindungen, z. B. Aminosäuren, Nucleinsäuren usw., auf der Erde durch die Einwirkung von UV-Strahlen, Glimmentladungen und Kernstrahlen auf die damals herrschende Atmosphare aus Wasser, Wasserstoff, Methan, Ammoniak, Kohlenoxyden, Schwefelwasserstoff usw. ausgelöst wurde $^{3)}$. Denn man konnte im Laboratorium durch Bestrahlen der einfachsten Ausgangsverbindungen eine

+) Von den Betrachtungen sollen exotherme Reaktionen ausgeschlossen werden, da diese meist als Kettenreaktionen ablaufen und dura Strahleneinwirkungen ebenso wie durch einmalige Zufuhr thermischer Energie oder durch Zugabe eines Katalysators induziert werden können; siehe z. B. die Athylbromid-Produktion aus Athylen und Bromwasserstoff, die durch $\gamma$-Strahlen induziert wird ${ }^{1}$ \}.
Reihe von Aminosäuren, Purinbasen usw. im Submikrogramm-Maßstab herstellen ${ }^{4}$.

Seit Strahlenquellen hoher Intensität zur Verfügung stehen, ist die Möglichkeit für strahlenchemische Reaktionen zur Erzeugung energiereicher chemischer Produkte auch im technischen Maßstabe gegeben. Ob solche Verfahren allerdings mit den altbekannten technischen Prozessen konkurrieren können, läßt sich heute noch nicht mit Sicherheit vorhersagen.

\section{Allgemeine Voraussetzungen}

An dem folgenden, bewußt ungünstig ausgewählten Beispiel soll abgeschätzt werden, von welchen Faktoren die Wirtschaftlichkeit strahlenchemischer Prozesse in technischem Maßstab abhängt und was für Produkte man herstellen kann. Es sei zunächst die Herstellung von Wasserst of $f$ nach Reaktion (1) durch Einwirkung von $\gamma$-Strahlen einer ${ }^{60} \mathrm{Co}$-Quelle auf Wasser betrachtet.

Der G-Wert**) für die Bildung von Wasserstoff ist etwa 0,5 Molekeln je $100 \mathrm{eV}^{\mathbf{s}}$ ), d. h. $200 \mathrm{eV}$ müssen für die Bildung einer Molekel oder $4600 \mathrm{kcal}$ für die Bildung eines Mols Wasserstoff aufgewandt werden $(1 \mathrm{eV}$ : Molekel $\approx 23 \mathrm{kcal} / \mathrm{Mol}$ ), wahrend die Wärmetönung der Reaktion nur $182,4 \mathrm{kcal} / \mathrm{Mol}$ ist. Der energetische Wirkungsgrad der Radiolyse des Wassers ist ebenso wie der der meisten anderen endothermen, durch Kernstrahlen ausgelösten Reaktionen sehr klein $(<5 \%)$. Warum das so ist, kann man leicht verstehen, wenn man die während der Bestrahlung ablaufenden Reaktionen kennt. Die Strahlung verliert durch Wechselwirkung mit den Elektronen der durchlaufenden Materie ihre Energie. Die Elektronen werden dabei, je

${ }^{++}$) Der G-Wert gibt an, wieviel Molekeln einer Substanz je $100 \mathrm{eV}$ im diemischen System absorbierter Energie gebıldet werden. 
nach der übertragenen Energie, auf höhere Energieniveaus gehoben oder ganz aus dem Atom- oder Molekelverband entfernt. Die herausgeschlagenen Elektronen können ihrerseits, wenn sie genügend Energie haben, weitere Anregungs- und Ionisierungsprozesse auslösen. Die absorbierte Energie wird dabei zu etwa gleichen Teilen zur Anregung und zur Ionisierung verbraucht. An diese energieverbrauchenden Primärreaktionen schließen sich die exothermen Folgereaktionen (Dunkelreaktionen) an, die zur Bildung neuer Molekeln führen.

Entsprechend der unterschiedlichen Wechselwirkungswahrscheinlichkeit der einzelnen Kernstrahlenarten mit den Elektronen der Materie unterscheiden sich die Strahlen in der je Weg-Einheit abgegebenen Energie und damit in der Dichte der in einer Strahlenspur erzeugten angeregten Molekeln und Ionen. Deshalb mag die Ausbeute einzelner Produkte von der Strahlenart abhängen, es werden dabei aber keineswegs verschiedenartige Reaktionen ablaufen oder verschiedenartige Produkte gebildet.

Der strahlenchemische Primärvorgang der Radiolyse des Wassers kann durch<smiles>O=CCC(C=O)NCCO</smiles>

beschrieben werden ${ }^{5,6}$. In den sich anschließenden Reaktionen entstehen $\mathrm{H}$ - und $\mathrm{OH}$-Radikale, die in verschiedener Weise rekombinieren und $\mathrm{H}_{2} \mathrm{O}_{2}$ und $\mathrm{H}_{2}$ bilden oder auch in Umkehrung ihrer Entstehung Wasser zurückbilden.

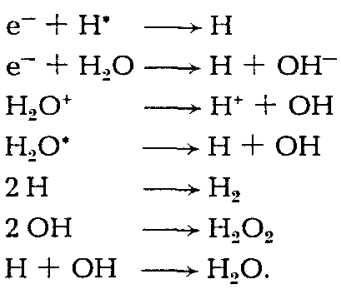

Leider geht ein Teil der Radikale durch die letzte Reaktion wieder verloren, wobei die in den Radikalen gespeicherte Energie in Wärme verwandelt wird. Aber auch die von den primär gebildeten, angeregten und ionisierten Molekeln aufgenommene Energie kann, ohne zu weiteren Reaktionen verbraucht $\mathrm{zu}$ werden, durch Desaktivierung als Wärme abgegeben werden:

$\mathrm{H}_{2} \mathrm{O}^{+}+\mathrm{e}^{-} \longrightarrow \mathrm{H}_{2} \mathrm{O}^{*}$

$\mathrm{H}_{2} \mathrm{O}^{*} \longrightarrow \mathrm{H}_{2} \mathrm{O}$.

Schließlich kann der entstandene Wasserstoff durch Angriff eines Radikals wieder zerstört werden:

$\mathrm{OH}+\mathrm{H}_{2} \longrightarrow \mathrm{H}_{2} \mathrm{O}+\mathrm{H}$.

Aus diesen Gründen, also wegen der unerwünschten Radikalrekombination, der Desaktivierung der angeregten Molekeln und der Zerstörung des Reaktionsproduktes durch weitere Strahleneinwirkungen sind die strahlenchemischen Prozesse vom energetischen Standpunkt aus nicht sehr ergiebig.

$4600 \mathrm{kcal}$, die zur Erzeugung $1 \mathrm{Mols}$ Wasserstoff durch $\gamma$-Strahlen aufgewandt werden müssen, entsprechen einer Energie von $5,3 \mathrm{kWh}\left(10^{3} \mathrm{kcal}=1,16 \mathrm{kWh}\right)$. Da die Energie der $\gamma$-Strahlen je nach Größe der Strah-
Tabelle 1. Strahlungskosten technischer Strahlenquellen

\begin{tabular}{|c|c|c|c|c|}
\hline $\begin{array}{l}\text { Strahlen- } \\
\text { quelle }\end{array}$ & $\begin{array}{l}\text { Strah- } \\
\text { Iung }\end{array}$ & Energie & $\begin{array}{l}\text { Strahlungs- } \\
\text { leistung }\end{array}$ & $\begin{array}{c}\text { Strahlungs- } \\
\text { kosten } \\
\text { DM/kWh }\end{array}$ \\
\hline $\begin{array}{l}\text { Beschleu- } \\
\text { niger }{ }^{7} \text { ) }\end{array}$ & $\begin{array}{l}\text { Elek- } \\
\text { tronen }\end{array}$ & $\begin{array}{l}\text { b1s } \\
10 \mathrm{MeV}\end{array}$ & 3 bis $100 \mathrm{~kW}$ & 34 bis 8 \\
\hline $\begin{array}{l}{ }^{60} \mathrm{Co}- \\
\text { Quelle }\end{array}$ & $\gamma$ & $\begin{array}{l}\text { 1,17 u. } \\
1,33 \mathrm{MeV}\end{array}$ & 3 bis $30 \mathrm{~kW}$ & 38 bis 13 \\
\hline $\begin{array}{l}{ }^{137} \mathrm{Cs}^{-} \\
\text {Quelle }\end{array}$ & $\gamma$ & $0,66 \mathrm{MeV}$ & 3 bis $30 \mathrm{~kW}$ & 46 bis 17 \\
\hline $\begin{array}{l}\text { Chemie- } \\
\text { Kern- } \\
\text { reaktory }\end{array}$ & $\begin{array}{l}\text { Neu- } \\
\text { tronen } \\
+\gamma\end{array}$ & $\begin{array}{l}\text { bis } \\
10 \mathrm{MeV}\end{array}$ & 1 bis $4 \mathrm{MW}$ & $0,5 \mathrm{~b} 1 \varepsilon 0,05$ \\
\hline $\begin{array}{l}\text { Chemie- } \\
\text { Kern- } \\
\text { reaktor }^{8} \text { ) }\end{array}$ & $\begin{array}{l}\text { Spalt- } \\
\text { bruch- } \\
\text { stucke }\end{array}$ & $85 \mathrm{McV}$ & bis $200 \mathrm{MW}$ & 0,1 bus 0,02 \\
\hline
\end{tabular}

lungsquelle 13 bis $38 \mathrm{DM} / \mathrm{kWh}$ kostet, Tabelle 1 , ist der so erzeugte Wasserstoff mit Kosten von 70 bis $200 \mathrm{DM}$ je Mol belastet. Wasserstoff, der durch Elektrolyse, Kohlenmonoxyd-Konvertierung oder aus Erdgas hergestellt wird, kostet nicht mehr als $0,5 \mathrm{Pfg}$./Mol, ist also wenigstens zehntausendmal billiger als der durch Radiolyse von Wasser mit $\gamma$-Strahlen erzeugte Wasserstoff.

Dieser bewußt ungünstig ausgewählte Vergleich soll zeigen, welche Bedingungen an die ökonomische Ausnutzung der Kernenergie zur Erzeugung energiereicher chemischer Produkte geknüpft sind. Zunächst muß eine billigere Strahlenquelle herangezogen werden

Um mit konventionellen Kraftwerken konkurrieren zu konnen, darf die in Kernkraftwerken erzeugte elektrische Energie mit nur zwei bis drei Pfennigen je kWh belastet sein. Folglich kann auch die Strahlenenergie in einem $\mathrm{Kern} \mathrm{r}$ e $\mathrm{kt}$ or, der als Großstrahlenquelle (Chemiereaktor) dienen kann, nicht teurer sein, Tabelle 1. Dabei kann man entweder die $\gamma$-Strahlen und schnellen Neutronen, also die sog. Reaktormischstrahlung, mit Energien bis zu $10 \mathrm{MeV}$, oder die sehr energiereichen Spaltbruchstücke, die aus dem gespaltenen Kern entstehen und eine Energie von fast $85 \mathrm{MeV}$ mit sich führen, ausnützen, Tabelle 2. Wie dies geschehen könnte, soll weiter unten gezeigt werden. Alle anderen Strahlungsquellen, wie Teilchenbeschleuniger oder natürlich radioaktive Nuclide, sind sicher zu teuer. Es bleibt abzuwarten, ob radioaktive Spaltprod u k t e, die als lästiges Nebenprodukt bei der Kernenergiegewinnung entstehen, in geeigneter Form zu so niederen Preisen angeboten werden können, daß sie als wirtschaftliche Strahlenquelle ausgenützt werden können.

Dann muß man sich auf ein Prod ukt spezialisieren, das in großen Mengen verbraucht wird, einen genügend

Tabelle 2. Verteilung der bei der Spaltung von ${ }^{23} \mathrm{U}$ mit langsamen Neutronen freiwerdenden Energle ${ }^{9}$ )

\begin{tabular}{lrr}
\hline Spaltbruchstücke, kin. Energıe & $168 \mathrm{MeV}$ & $83,5 \%$ \\
Neutronen, kin. Energie & $5 \mathrm{MeV}$ & $2,5 \%$ \\
prompte $\gamma$-Strahlung & $5 \mathrm{MeV}$ & $2,5 \%$ \\
$\gamma$-Strahlung der Spaltbruchstücke & $6 \mathrm{MeV}$ & $3,0 \%$ \\
$\beta$-Strahlung der Spaltbruchstücke & $7 \mathrm{MeV}$ & $3,5 \%$ \\
Neutrinos & $10 \mathrm{MeV}$ & $5,0 \%$ \\
\hline Insges. je Spaltung freiwerdende Energie & $201 \mathrm{MeV}$ & \\
\hline
\end{tabular}


hohen Wert besitzt und schließlich auch mit hoher Strahlenausbeute aus billigen Ausgangsstoffen hergestellt werden kann.

Auf strahlenchemischem Wege Verbindungen komplizierterer Struktur herzustellen, ist sehr unökonomisch, da die Bildungswahrscheinlichkeit oder Ausbeute solcher Molekeln außerordentlich gering ist. Denn bei strahlenchemischen Prozessen muß man es meist dem Zufall überlassen, wie die primär gebildeten Radikale oder Ionen reagieren. Die Bildung komplizierter Molekeln ist dabei aus statistischen Gründen unwahrscheinlich. Demnach scheint vom wirtschaftlichen Standpunkt aus nur die strahlenchemische Synthese e in $\mathrm{f} \mathrm{a} \mathrm{ch}$ er, aber wertvoller Verbindungen in einem Kernreaktor unter Ausnutzung der Energie der Reaktormischstrahlung oder der Spaltfragmente interessant. Als Ausgangsstoffe kommen dabei allerdings keine Stoffe in Frage, die infolge eines großen Neutroneneinfangquerschnittes ihrer Elemente die NeutronenOkonomie des Reaktors gefährden und gleichzeitig wegen des Neutroneneinfangs stark radioaktive Verbindungen bllden. Dadurch wird die Anwendbarkeit eines Kernreaktors für strahlenchemische Reaktionen beschränkt auf Ausgangsmaterialien, die hauptsächlich Wasserstoff, Kohlenstoff, Stickstoff und Sauerstoff enthalten. Das ist aber kein großer Nachteil, da ein wesentlicher Teil der Grundchemikalien der chemischen Industrie nur aus Stoffen mit den genannten Elementen besteht: Wasser, Ammoniak, Kohlenoxyde, Stickoxyde, Kohlenwasserstoffe und ihre Oxydationsprodukte, Amine usw.

\section{Aussichtsreiche strahlenchemische Synthesen}

Gegenwártig werden strahlenchemische Prozesse zur großindustriellen Erzeugung von Glykol, Phenol, Stickstoffdioxyd, Kohlenmonoxyd und Hydrazin diskutiert ${ }^{10,11}$, Tabelle 3. Diese Substanzen und die dazugelörigen Ausgangschemikalien erfüllen die Voraussetzungen, die vorher genannt worden sind.

Die Phenol-Synthese, die als schwach exotherme Reaktion verlauft, ist eigentlich kein Beispiel für die Umwandlung von Kernenergie in chemische Energie. Sie ist hier jedoch zum Vergleich aufgeführt. Ihr hoher G-Wert weist auf eine Kettenreaktion hin, ähnlich wie sie bei der $\gamma$-strahleninduzierten Polymerisation von Acetylen $(G=75)$ oder Äthylen $(G=6500)$ oder bei der strahlenchemischen Chlorierung von Benzol zu Hexachlorocyclohexan $(G=85000)$ beobachtet wird.
Die strahlenchemische $\mathrm{Hydraz}$ in - Synthese erscheint zunächst wegen des gegenwärtigen hohen Hydrazin-Preises (etwa $10 \mathrm{DM} / \mathrm{kg}$ ) vom wirtschaftlichen Standpunkt aus besonders attraktiv. Es ist aber mit Sicherheit anzunehmen, daß bei größerem Bedarf an Hydrazin (z. B. als Treibmittel für Raketen) billigere Produktionsverfahren entwickelt werden, die die alte, über Chloramin verlaufende Synthese nach Raschig verdrängen werden. Das heißt, daß ein wegen des jetzigen hohen Hydrazin-Preises wirtschaftliches strahlenchemisches Verfahren zur Hydrazin-Synthese nicht unbedingt auch in der Zukunft von wirtschaftlichem Vorteil sein muß. - Diese Einschränkungen gelten nıcht für die anderen in Tab. 3 aufgeführten Stoffe, da bei diesen wegen der ohnehin niedrigen Preise nicht mit neuen, stark kostensenkenden Verfahren gerechnet zu werden braucht.

Im folgenden sollen die Grundlagen sowie die technischen und wirtschaftlichen Aspekte der chemonuclearen Glykol-Synthese und der Stickstoff-Fixierung in einem Chemie-Kernıeaktor unter Ausnutzung der Energie der Reaktormischstrahlung bzw. der kinetıschen Energie der Spaltprodukte besprochen werden.

\section{Reaktionen bei der Bestrahlung von Methanol und Stickstoff/Sauerstoif-Mischungen}

Die Reaktionen, die bei der Bestrahlung von Methanol und Stickstoff/Sauerstoff-Mischungen ablaufen, sind wegen der Vielzahl der möglichen Reaktionsprodukte unübersichtlicher als die Radiolyse des Wassers; denn außer den gewunschten Produkten entstehen eine Anzahl weiterer Verbindungen wie Methan, Formaldehyd, Ameisensaure, hohere Polymere, Kohlenmonoxyd und Kohlendioxyd im ersten Falle und die Stickoxyde vom Distickstoffoxyd bis zum Stickstoffpentoxyd und Ozon im zweiten Falle. Deshalb sind die Mechanismen der Reaktionen, die zum Glykol bzw. Stickoxyd führen, noch nicht sicher aufgeklärt.

Der bei der Synthese von Glykol aus flüssigem Methanol diskutierte Mechanismus ${ }^{27,28)}$ schliebt sich eng an den fur die Radiolyse des Wassers an: die primär ionisierten und angeregten Methanol-Molekeln dissoziieren und bilden $\mathrm{CH}_{2} \mathrm{OH}$-Radikale und $\mathrm{H}$-Atome, die zu Glykol und Wasserstoff rekombinieren. Ein einzelnes H-Atom kann durch Reaktion mit einer Methanol-Molekel ein weiteres $\mathrm{CH}_{2} \mathrm{OH}$-Radikal bilden, das auch zur Glykol-Bildung zur Verfugung steht:

Tabelle 3. Aussichtsreiche strahlenchemische Reaktionen im Kernreaktor

\begin{tabular}{|c|c|c|c|c|c|}
\hline Produkt & Glykol12-14) & Phenol $\left.{ }^{13,15}\right)$ & Hydrazın $\left.{ }^{16-19}\right)$ & Stickstoffdioxyd $20-25)$ & Kohlenmonoxyd 21 .6 \\
\hline Ausgangsstoffe & $\begin{array}{l}\text { flussig. } \\
\text { Methanol }\end{array}$ & $\begin{array}{l}\text { Benzol }+ \text { Luft } \\
\text { in Wasser }\end{array}$ & flussıg. Ammonıak & Luft & $\begin{array}{l}\text { Kohlendıoxyd } \\
\text { mit } \mathbf{1}^{0 / 0 \mathrm{NO}_{2}}\end{array}$ \\
\hline $\begin{array}{l}\text { Summen- } \\
\text { reaktion }\end{array}$ & $\begin{array}{l}2 \mathrm{CH}_{3} \mathrm{OH} \rightarrow \\
\left(\mathrm{CH}_{2} \mathrm{OH}\right)_{2}+\mathrm{H}_{2}\end{array}$ & $\begin{array}{l}\mathrm{C}_{6} \mathrm{H}_{3}+1 / 2 \mathrm{O}_{2} \rightarrow \\
\mathrm{C}_{6} \mathrm{H}_{5} \mathrm{OH}\end{array}$ & $2 \mathrm{NH}_{3} \rightarrow \mathrm{N}_{2} \mathrm{H}_{4}+\mathrm{H}_{2}$ & $1 / 2 \mathrm{~N}_{2}+\mathrm{O}_{2} \rightarrow \mathrm{NO}_{2}$ & $\mathrm{CO}_{2} \rightarrow \mathrm{CO}+1 / 2 \mathrm{O}_{2}$ \\
\hline $\begin{array}{l}\text { Reaktionswárme } \\
\text { [kcal/Mol] }\end{array}$ & 3,6 & $-1,9$ & 48 & 8,1 & 67,6 \\
\hline$G_{y+N}$ & 3 & $>30$ & 0,1 & 5 & - \\
\hline$G_{\text {Spaltfragmente }}$ & 1 & - & 1,9 & 7,5 & 10 \\
\hline
\end{tabular}




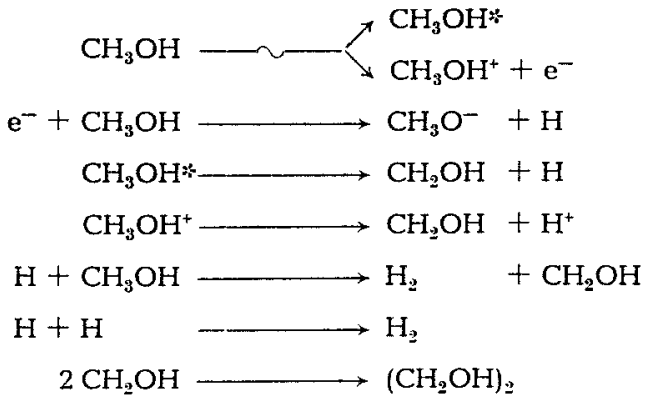

Da der Temperaturkoeffizient der Geschwindigkeit der Reaktion eines Wasserstoff-Atoms mit einer MethanolMolekel größer ist als der der Wasserstoff-Rekombination ${ }^{\mathbf{1 2})}$, wird die erstere gegenuber der zweiten bei höheren Temperaturen begünstigt. Deshalb ist eine erhöhte Temperatur zur Erzielung größerer GlykolAusbeuten bei der Bestrahlung von Methanol vorteilhaft.

Die Stickstoff-Fixierung wird eingeleitet durch Anregung einer Stickstoff-Molekel und anschließende Dissoziation in Stickstoff-Atome ${ }^{20,21}$. Diese setzen sich mit Sauerstoff-Molekeln zu Stickstoffmonoxyd und weiter zu Stickstoffdioxyd um, das in einer Gleichgewichtsreaktion Stickstofftetroxyd bildet. - Der Angriff von Stickstoff-Atomen auf Stickstoffdioxyd, der teilweise zu molekularem Stickstoff, dem Ausgangsstoff, führt, bedeutet eine storende Ausbeuteverminderung der Stickstoff-Fixierung.

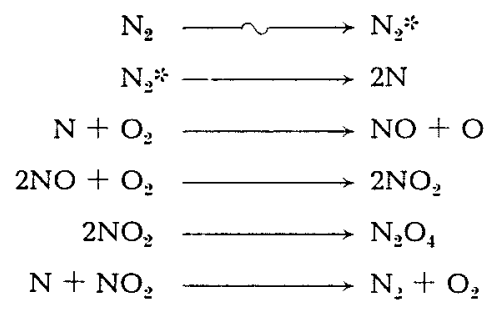

Der Abbau des durch strahlenchemische Reaktionen gebildeten Produkts bei weiterer Strahleneinwirkung ist immer zu befürchten und wegen der damit verbundenen Ausbeuteverminderung unerwünscht. Da der Abbau natürlich mit steigender Konzentration des Produktes im Strahlungsraum immer schneller wird, muß man durch laufende Abtrennung des gewünschten Produktes seine Konzentration so niedrig halten, daß die Abbaureaktionen die Ausbeute nicht mehr merklich verringern. Dies geschieht bei der Stickstoff-Fixierung sehr leicht, da bei Anwendung höherer Drucke Stickstoffdioxyd in Stickstofftetroxyd ubergeht, das auskondensiert und damit aus der Gasphase ausscheidet.

Im angegebenen Reaktionsschema verläuft die Stickstoff-Fixierung ausschließlich ủber primär gebildete Stickstoff-Atome. Sauerstoff-Atome, die durch strahleninduzierte Dissoziation des molekularen Sauerstoffs oder in anderen Sekundärreaktionen entstehen, können Stickstoff-Molekeln nicht aufspalten und haben damit keinen Einfluß auf die Ausbeute bei der StickstoffFixierung. Das ist bedingt durch die extreme Stabilitat der Stickstoff-Molekel.

Das Reaktionsschema berücksichtigt nicht die Ionenreaktionen, die neben den Radikalreaktionen ablaufen und auch zur Stickstoff-Fixierung beitragen (wie z. B.
$\mathrm{N}_{2}^{+}+\mathrm{O}_{2} \longrightarrow \mathrm{NO}+\mathrm{NO}^{+}$) und läBt außerdem die Bildung der anderen Stickoxyde auBer acht. Das Schema ist also bei weitem nicht vollkommen. Einige Aussagen über die günstigsten Bedingungen bei der StickstoffFixierung lassen sich aber doch daraus ableiten ${ }^{21}$. Da bei Drucksteigerung das Gleichgewicht zwischen Stickstoffdioxyd und Stickstofftetroxyd zugunsten des Tetroxyds, das auskondensiert, verschoben wird, ist ein hoher Druck im Strahlungsraum vorteilhaft. Weiterhin ist ein Sauerstoff-UberschuB günstig, da damit die Wahrscheinlichkeit der Reaktion der Stickstoff-Atome mit Sauerstoff-Molekeln gegenüber der mit Stickstoffmonoxyd oder Stickstoffdioxyd, also die Aufbaureaktion gegenüber der Abbaureaktion, begünstigt wird. Den gleichen Effekt hat auch eine erhöhte Temperatur (z. B. $>250^{\circ} \mathrm{C}$ ), da die Aktivierungsenergie der Aufbaureaktion größer ist als die der Abbaureaktion.

Die Stickstoff-Fixierung würde man vorteilhafter unter Ausnützung der Rückstoßenergie der Spaltfragmente durchfuhren. Bei der Glykol-Synthese wurde man dagegen die Reaktormischstrahlung heranziehen, da die schon verhältnismäßig komplizierte Methanol-Molekel gegen die Rückstoß-Spaltkerne sehr viel anfälliger ist als gegen die Reaktormischstrahlung. Denn bei der durch die Rückstoß-Spaltfragmente induzierten GlykolSynthese werden je gebildete Glykol-Molekel nahezu zehn Methanol-Molekeln unter Bildung von Kohlenmonoxyd, Methan und Formaldehyd zerstòrt, während bei der Reaktormischstrahlung außer den zur GlykolBildung verbrauchten zwei Methanol-Molekeln nur ein weiteres zerstört wird ${ }^{\mathbf{1 4}}$ ).

\section{Möglicher Aufbau von Chemie-Kernreaktoren}

Ausnutzung der Reaktormischstrahlung

Wenn die Reaktormischstrahlung zur Durchführung der dhemischen Synthesen verwendet werden kann, dürften sich über die üblichen Probleme des Reaktorbaus hinaus keine wesentlichen technischen Schwierigkeiten ergeben. Hierbei wird allerdings nur ein geringer Teil der Reaktorenergie ausgenutzt, denn je Spaltung werden nur etwa $8 \%$ der gesamten Spaltenergie von $200 \mathrm{MeV}$ als $\gamma$-Strahlen und schnelle Neutronen frei, Tabelle 2; der Hauptanteil, nämlich nahezu $85 \%$ der Spaltenergie, entfällt auf die kinetische Energie der Spaltfragmente, die vollständig in den Brennelementen absorbiert wird. Von den $8 \%$ Mischstrahlung steht uns höchstens die Hälfte, also etwa $4 \%$ der Gesamtenergie zur Verfügung. Der Rest wird in den Brennelementen selbst, im Kühlkreislauf, von den Reaktor-Konstruktionsmaterialien und evtl. im Neutronen-Moderator absorbiert.

Kerntechnisch und konstruktiv könnte jeder organisch moderierte Reaktor bereits als Chemie-Kernreaktor für die Synthese organischer Verbindungen verwendet werden. In organisch moderierten Reaktoren ist z. B. Diphenyl als Moderator vorgesehen, eine gegen Kernstrahlung außerordentlich resistente Verbindung. Ohne wesentliche Abänderung des Reaktors, d.h. ohne getrennte Bestrahlungskanäle einzubauen, könnte man das Diphenyl gegen flüssiges Methanol oder andere flüssige organische Systeme austauschen und damit im Moderatorkreislauf die angegebenen Synthesen ablau- 
fen lassen. In enger Anlehnung an die technischen und betrieblichen Erfahrungen, die die Atomics International mit dem OMR (organisch moderiertem Reaktor) gemacht hat, wurde von derselben Firma ${ }^{29}$ ) eine $1 \mathrm{MW}$ Versuchsanlage projektiert, in der $3,5 \%$ der thermischen Leistung des Reaktors als Reaktormischstrahlung im chemischen Reaktionsgemisch absorbiert werden.

Das Reaktionsgemisch wird dabei an den Brennelementen vorbeigeleitet, aber von diesen durch den Kühlmittelkreislauf getrennt. Die Konstruktion eines solchen Chemie-Kernreaktors folgt aus Abb. 1. Das einzelne Brennstoffelement ist aus konzentrischen Zylindern einer Uran-Legierung mit auf 3,5\% angereichertem ${ }^{235} \mathrm{U}$ aufgebaut, die mit Aluminium verkleidet sind. Durch die zylindrischen Spalten wird das Kühlmittel, Kohlendioxyd oder Diphenyl, gepumpt, während das Reaktionsgemisch durch das Zentrum und am Außenumfang des Brennelementes vorbeifließt. 19 solcher Brennelemente sind im Core von $60 \mathrm{~cm}$ Höhe und $70 \mathrm{~cm}$ Durchmesser zwischen zwei Tragplatten angeordnet. Der Materialfluß und Kühlmittelfluß sind aus der Abbildung zu entnehmen.

Ausnutzung der Ruckstoßenergie

der Spaltragmente

Ganz andersartige Konstruktionsprinzipien müssen gewählt werden, wenn die Ruckstoßenergie der Spaltfragmente, die $85 \%$ der Gesamtspaltenergie ausmacht, ausgenutzt werden soll; denn ihre Reichweite, die in flüssigen und festen Medien kleiner als $30 . \mathrm{m}$ ist, verbietet die Verwendung von kompakten Brennelementen. Der Brennstoff muß vielmehr ohne Verkleidung in feinster Verteilung im chemischen Reaktionsraum untergebracht werden. Die ideale Form scheint zunächst die homogene, molekulardisperse Verteilung zu sein, also Anwendung von gasförmigem Uranhexafluorid, das bei Gasreaktionen (z. B. der Stickstoff-Fixierung) der Gasphase zugesetzt wird, oder von löslichen UranVerbindungen bei Reaktionen in flussiger Phase (z. B. Urannitrat bei der Hydrazin-Synthese in flussigem Ammoniak). Unter diesen Bedingungen könnte die Rückstoßenergie der Spaltfragmente vollständig im chemischen Reaktionsgemisch absorbiert werden. Da aber die genannten Uran-Verbindungen sehr wenig strahlenresistent sind, ist schon nach kurzem Betrieb mit der Ausscheidung fester Uran-Verbindungen zu rechnen. Außerdem durfte die erforderliche kontinuierliche $\mathrm{Ab}$ trennung des Kernbrennstoffs vom chemischen Reaktionssystem ziemlich aufwendig sein. Deshalb ist es vorteilhafter, selbst unter Verlust eines Teils der Rückstoßenergie ein heterogenes System von fein verteiltem Brennstoff und chemischem Reaktionsgemisch vorzusehen.

Die hierfür diskutierten Reaktorformen sind der Festbett-Reaktor und der Staubphasen-Reaktor. Als Festbett-Reaktor hat wahrscheinlich der $\mathrm{Glas}$ faser$\mathrm{Reaktor} \mathrm{r}^{21,30,31)}$ die größte Chance. Hier soll bis zu $50 \%$ hochangereichertes Uranoxyd enthaltende Glaswolle mit einem Durchmesser von weniger als $5 \mu \mathrm{m}$ als Brennstoff verwendet werden. Die Glasfaser soll fest im Reaktorcore eingebaut und vom flüssigen oder gasförmigen Reaktionsgemisch umströmt werden. Mit dieser Anordnung sollen bis zu 50\% der Rückstoßenergie
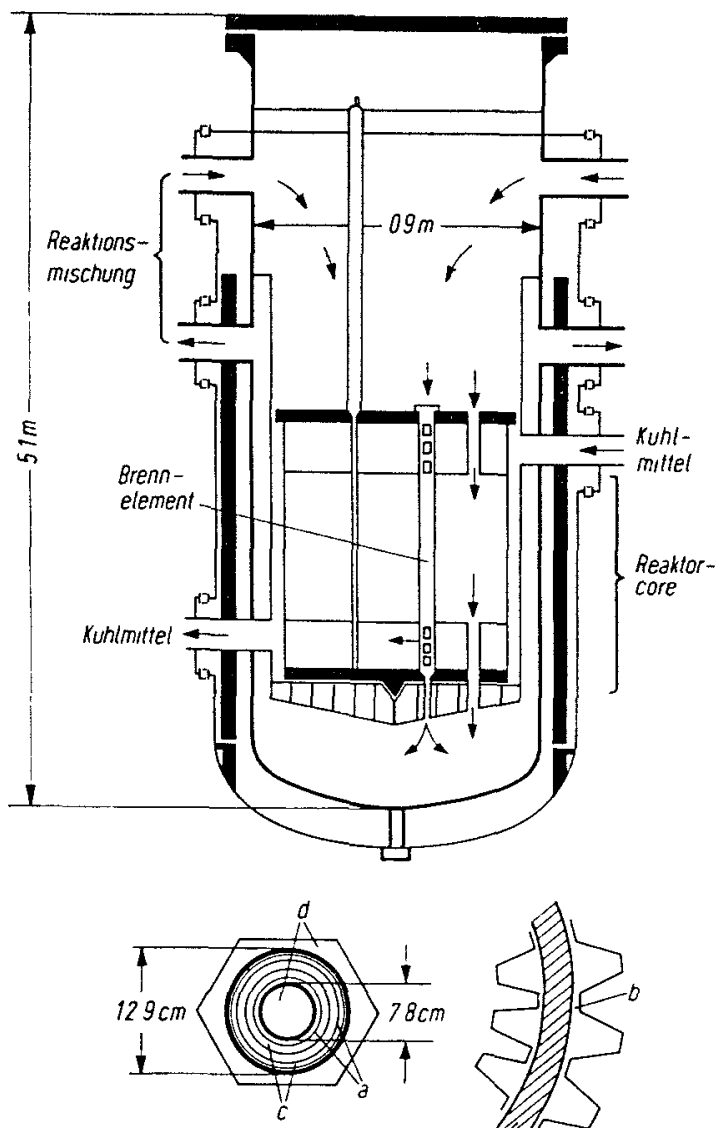

51657

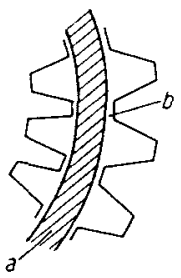

Abb. 1. Querschnitt durch einen Cheme-Kernreaktor zur Ausnutzung der Reaktormischstrahlung (schematisch)

Darunter: Einzelheiten eines Brennelementes ${ }^{29}$ ).

a Uran-Molybdan-Alumınium-Legıerung, $b$ Alumınım-Umhullung, $c$ Kuhlmittel, $d$ Reaktionsmischung

der Spaltfragmente für chemische Zwecke zur Verfúgung stehen.

Beim Sta ub hasen-Reaktor wird vorausgesetzt, daß das Brennmaterial als Uranoxyd in Staubform mit Partikeln von Durchmessern bis herab zu $1 \mu \mathrm{m}$ eingesetzt und vom Strom des gasförmigen oder flussigen Reaktorgemischs aufgewirbelt und getragen wird ${ }^{24,32,33)}$. Hierbei sollen bis zu 95\% der Spaltfragmentenergie ausgenutzt werden können.

Ein vom Brookhaven National Laboratory geplanter Staubphasen-Reaktor ${ }^{33}$ ) sieht ein zylindrisches Core von $2,5 \times 2,5 \mathrm{~m}$ vor, das vom Graphit-Reflektor umgeben ist, Abb. 2. Die Reaktorumhüllung ist fùr einen Gasdruck bis zu 100 at ausgelegt. Das Innere des Core ist zu 80\% mit langgezogenen Graphit-Blöcken (Moderator) ausgefullt. Von unten tritt das gasförmige Reaktionsgemisch, bei der Stickstoff-Fixierung eine Stickstoff/Sauerstoff-Mischung, im einfachsten Falle Luft, unter einem Druck von 100 at zusammen mit dem staubförmigen Brennstoff ein; oben ist die Austrittsoffnung. Die Kühlung des Reaktors wird hier, wie auch beim Glasfaser-Reaktor, von den Reaktionsgasen übernommen. Gaseintrittstemperatur ist $290^{\circ} \mathrm{C}$, die Austrittstemperatur $600^{\circ} \mathrm{C}$. 


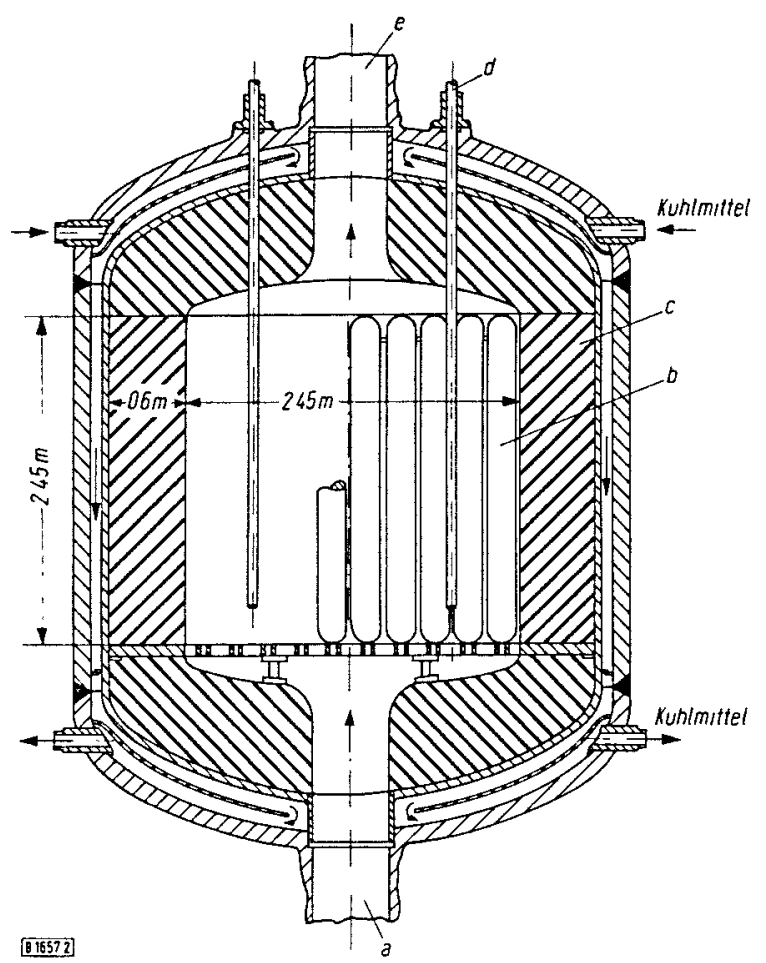

Abb. 2. Querschnitt durch ennen Chemie-Kernreaktor zur Ausnutzung der Ruckstoßenergle der Spaltfragmente. Als Kermbrennmaterial ist Urandioxyd-Staub vorgesehen ${ }^{33}$ ).

$a$ Eintritt fur Reaktionsmischung und Brennstoff-Staub, $b$ Graphit-Moderator, c Graphit-Reflektor, $d$ Kontroll-Stab, e Austritt fur Reaktionsprodukt und Brennstoff-Staub.

Vergleich der Chemie-Kernreaktortypen

Aus der Gegenüberstellung der zwei Chemie-Kernreaktortypen, die die Energie der Reaktormischstrahlung bzw. der Rückstoßenergie der Spaltfragmente ausnutzen, können wir leicht ihre Vor- und Nachteile ableiten: im ersten Reaktortyp steht nur ein kleiner Teil, nämlich bis zu $4 \%$ der gesamten Reaktorenergie zur Absorption im chemischen System zur Verfügung. Abgesehen von der Aktivierung durch Neutronen ist das Reaktionsprodukt aber sauber. Im zweiten Reaktortyp können bis zu $80 \%$ der Reaktorenergie im chemischen System absorbiert werden. Der Wirkungsgrad dieses Reaktors für chemische Synthesen ist also sehr viel größer. Da aber die Spaltfragmente im chemischen Produkt eingefangen werden, ist dieses hochgradig radioaktiv, und zu seiner Reinigung müssen ähnliche Anlagen erstellt werden wie zur Aufarbeitung von Reaktorbrennstoffen.

Der Festbett- und der Staubphasen-Reaktor haben ebenfalls ihre gegeneinander abzuwägenden Vor- und Nachteile, die wir am Beispiel der Stickstoff-Fixierung diskutieren wollen. Ein Festbett-Reaktor ist bei stórungsfreiem Betrieb leichter zu kontrollieren als ein Staubphasen-Reaktor, bei dem Inhomogenitäten der Staubphase leicht zu Reaktivitätsänderungen des Reaktors führen können. Außerdem tritt im StaubphasenReaktor der staubförmige Brennstoff zusammen mit dem Reaktionsgut an der Entnahmeseite aus und muß von diesem mechanisch, z. B. durch Zyklone, abgetrennt und den reinen Reaktionsgemischen wieder zugesetzt werden. - Wenn die Spaltfragmente ihre Energie mög- lichst vollständig an das gasförmige Reaktionsgemisch abgeben sollen, muß ihre Reichweite im Gas kleiner sein als der mittlere Abstand zweier Staubteilchen oder Glasfasern, da sonst ein Teil der Energie wieder im Brennstoff absorbiert wird. Das kann man durch Anwendung genügend hoher Gasdrucke, z. B. 100 at, erreichen. Da hierbei bis zu $7 \%$ der bei der Spaltung frei werdenden Neutronen vom Stickstoff eingefangen werden (n,p-Reaktion), setzt die Regelung des Reaktors eine in engen Grenzen gleichbleibende Stickstoff-Konzentration voraus. Wenn durch eine Undichtigkeit im Gaskreislauf der Stickstoff-Druck plötzlich abnimmt, kann ein Festbett-Reaktor durch die plötzliche Reaktivitätssteigerung außer Kontrolle geraten. - Beim Staubphasen-Reaktor ist dagegen bei einem Bruch im Leitungssystem damit zu rechnen, daß gleichzeitig mit dem Gas ein Teil des Brennstoffstaubes mit herausgerissen und damit eine gefährliche Reaktivitätssteigerung verhütet wird.

Bei den geplanten Chemie-Kernreaktoren kann nur ein Teil, nämlich je nach Typ 4 bis $80 \%$, der gesamten Spaltenergie ins chemische System eingestrahlt werden. Der Rest geht durch Absorption in den Brennelementen, den Reaktormaterialien usw. verloren. Von der im chemischen System absorbierten Strahlenenergie wird - wie am Beispiel der Radiolyse des Wassers gezeigt worden ist - wiederum nur ein kleiner Teil in chemische Energie umgewandelt. Trotzdem ist ein solcher Reaktor für chemonucleare Reaktionen nicht unbedingt unwirtschaftlich. Denn die verbleibende thermische Energie kann zur Erzeugung elektrischer Energie weitergegeben werden. Die Hauptkosten der Strahlung können damit vom chemischen Produkt abgewälzt werden, sie müssen vom Stromverbraucher mitgetragen werden. Deshalb werden bei den folgenden Kostenabschätzungen nicht die Investitionskosten des Kernreaktors allein, sondern die einer vollständigen Energieerzeugungsanlage eingesetzt.

Obwohl nach den vorhergehenden Betrachtungen das chemische Produkt vom energetischen Standpunkt aus nur ein Nebenprodukt der Energieerzeugung darstellt, dürfte ein Chemie-Kernreaktor doch nur wirtschaftlich arbeiten, wenn der Wert des erzeugten chemischen Produktes mit dem Wert der gleichzeitig erzeugten Energie vergleichbar ist.

\section{Abschätzung der Größe der Chemie-Kernreaktoren und der Kosten der chemischen Produkte}

\section{Glykol-Synthese}

Chemische Fabriken konventioneller Art, die Glykol aus Athylen, Athylenoxyd oder Dichloräthylen herstellen, haben eine Jahreskapazität von 10000 bis 50000 t. Für die weiteren Betrachtungen (Tab.4) soll eine Kapazität von $10000 \mathrm{t} / \mathrm{Jahr}$ zugrunde gelegt werden. Der G-Wert der Glykol-Synthese aus Methanol mit der Reaktormischstrahlung ist etwa 3 Molekeln je $100 \mathrm{eV}$, d. h. $33 \mathrm{eV}$ müssen je entstandener GlykolMolekel von Methanol absorbiert werden. Das entspricht einer Energie von $14,2 \mathrm{kWh} / \mathrm{kg}$. Im Jahr mit 8000 Betriebsstunden sollen $10000 \mathrm{t}$ Glykol hergestellt werden; die dazu benötigte Leistung ist $14,2 \mathrm{kWh} / \mathrm{kg} X$ $10^{7} \mathrm{~kg} / 8000 \mathrm{~h}=17,75 \mathrm{MW}$. Von der Gesamtenergie des 
Reaktors mögen $4 \%$ in Form der Mischstrahlen zur Synthese zur Verfügung stehen, so daß der Reaktor zur Produktion von $10000 \mathrm{t}$ Glykol pro Jahr eine Leistung von etwa $450 \mathrm{MW}$ (th) haben sollte. Das ist eine durchaus realisierbare Größe.

Nach amerikanischen Angaben ${ }^{34}$ ) aus dem Jahre 1961 belaufen sich die Investitionskosten für einen Kernreaktor mit angeschlossenem Kraftwerk mittlerer Größe auf 700 bis 1200 DM je kW(e). Hier soll der fur deutsche Verhältnisse sicher nicht zu pessimistische Preis von $1400 \mathrm{DM} / \mathrm{kW}(\mathrm{e})$ eingesetzt werden, in dem eventuelle Sonderheiten des Chemie-Kernreaktors schon berücksichtigt sein sollen.

Ein $450 \mathrm{MW}$ (th)-Reaktor, der bei einer gunstig angenommenen 30proz. Energieumwandlung $135 \mathrm{MW}(\mathrm{e})$ liefert, würde daher etwa 190 Mill. DM kosten. Für die angeschlossene chemische Fabrik zur Reindarstellung des Glykols sollen 10 Mill. DM angenommen werden. Jährliche Amortisation, Versicherung und Steuern mussen mit $15 \%$ der Investitionskosten angesetzt werden. Die Kosten für den laufenden Betrieb, das sind die Kosten für Brennelemente und ihre Wiederaufarbeitung, Unterhaltung und Reparaturen, Lohne und Aufarbeitung des chemischen Reaktionsproduktes werden ebenfalls mit $15 \%$ der Investitionskosten veranschlagt, so daß der jährliche Betrieb 60 Mill. DM kostet. Dazu kommen etwa 3 Mill. DM für die $15000 \mathrm{t}$ Methanol $(0,2 \mathrm{DM} / \mathrm{kg})$, die bei der Synthese von $10000 \mathrm{t}$ Glykol verbraucht werden.

Da durch die chemischen Prozesse nur etwa $1 \%$ der Gesamtenergie des Reaktors verbraucht, wird, stehen noch $445 \mathrm{MW}$ thermischer Energie oder bei der angenommenen 30proz. Umwandlung noch $134 \mathrm{MW}$ elektrischer Energie zur Verfügung. Bei 8000 Betriebsstunden pro Jahr können damit $1,07 \cdot 10^{9} \mathrm{kWh}$ abgegeben werden, die bei einem Preis von $3 \mathrm{Pfg}$. je $\mathrm{kWh}$ nahezu 33 Mill. DM einbringen. Damit belaufen sich die Kosten für die Produktion von $10000 \mathrm{t}$ Glykol auf 30 Mill. DM oder 3,00 DM je kg. Da die Gestehungskosten des Glykols nach konventionellen Produktionsmethoden etwa sechsmal niedriger sind, ist das diskutierte Verfahren zur Glykol-Synthese nach dem jetzigen Stand der Technik nicht konkurrenzfähig. - Das folgt auch bereits daraus, daB der tatsächliche Wert des erzeugten Glykols nur etwa 15\% des Wertes der elektrischen Energie ausmacht (5 gegenüber 33 Mill. DM).

\section{Stickstoff-Fixierung}

Wie durch Tab. 4 gezeigt werden soll, sind die ökonomischen Aspekte für die chemonucleare StickstoffFixierung günstiger. Hierbei soll die Rückstoßenergie der Spaltfragmente ausgenutzt werden. Entsprechend einem G-Wert von 7 für die Stickstoffdioxyd-Bildung aus Luft müssen $8,3 \mathrm{kWh}$ Strahlung je $\mathrm{kg}$ Stickstoffdioxyd absorbiert werden. Wenn eine Produktionskapazität von $500000 \mathrm{t}$ pro Jahr angenommen wird, wird hierzu eine Strahlungsleistung von $520 \mathrm{MW}$ benòtigt. Da bei Ausnützung der Rückstoßenergie der Spaltfragmente etwa $70 \%$ der gesamten Reaktorenergie zur Verfügung stehen, muß der Chemie-Kernreaktor für eine Leistung von $740 \mathrm{MW}$ (th) ausgelegt werden, was bei einem optimalen Wirkungsgrad von
Tabelle 4. Abschätzung der Größe von Chemie-Kernreaktoren und der Herstellungskosten der darin erzeugten Produkte

\begin{tabular}{|c|c|c|}
\hline Produkt & Glykol & Stıckstoffdioxyd \\
\hline Ausgangsstoff & Methanol & Luft \\
\hline Strahlung & $\begin{array}{l}\gamma-\text {-n-Misch- } \\
\text { strahlung }\end{array}$ & Spaltfragmente \\
\hline Jahreskapazitat & $10000 t$ & $500000 \mathrm{t}$ \\
\hline$G$ (Produkt) & 3 Molek $/ 100 \mathrm{eV}$ & 7 Molek. $/ 100 \mathrm{eV}$ \\
\hline Absorbierte Energie & $\begin{array}{l}33 \mathrm{eV} / \mathrm{Molek} \\
14,2 \mathrm{kWh} / \mathrm{kg}\end{array}$ & $\begin{array}{l}14,3 \mathrm{eV} / \text { Molek } \\
8.3 \mathrm{~kW} / \mathrm{kg}\end{array}$ \\
\hline $\begin{array}{l}\text { Absorbierte } \\
\text { Strahlenleistung }\end{array}$ & $17,75 \mathrm{MW}$ & $520 \mathrm{MV}$ \\
\hline Strahlenausnutzung & $4^{n / 6}$ & $70^{6 ;}, \mathrm{u}$ \\
\hline $\begin{array}{l}\text { Reaktorleistung bel } \\
\text { 30proz. Wirkungsgrad }\end{array}$ & $\begin{array}{l}450 \mathrm{MW}(\mathrm{th}) \\
135 \mathrm{MW}(\mathrm{e})\end{array}$ & $\begin{array}{l}740 \mathrm{MW}(\mathrm{th}) \\
222 \mathrm{MW}(\mathrm{e})\end{array}$ \\
\hline \multicolumn{3}{|l|}{ Investitionskosten } \\
\hline $\begin{array}{l}\text { Reaktorkraftwerk, } \\
\text { gesamt }\end{array}$ & 190 Mıll. DM & 265 Mill. DM \\
\hline Chem. Fabrik & 10 Mill DM & 100 Mill. DM \\
\hline Gesamt & 200 Mill. DM & 365 Mill. DM \\
\hline \multicolumn{3}{|l|}{ Jahrliche Belastung } \\
\hline $\begin{array}{l}\text { (Amortisation, Ver- } \\
\text { sicherung, Steuer, } \\
\text { Betriebskosten, Per- } \\
\text { sonalkosten, } \\
\text { Verwaltung) }\end{array}$ & G0 Mill. DM & 110 Mill DM \\
\hline Ausgangsproduht & 3 Mill. DM & - \\
\hline Gesamt & 63 Mill DM & 110 Mill. DM \\
\hline $\begin{array}{l}\text { Abgegebene } \\
\text { elektr. Energie }\end{array}$ & $1,1 \cdot 10^{7} \mathrm{kWh}$ & $1,7 \cdot 10^{3} \mathrm{kWh}$ \\
\hline Gutschr. $0,03 \mathrm{DM} / \mathrm{kWl}$ & 33 Mill. DM & $52 \mathrm{MJll} . \mathrm{DM}$ \\
\hline $\begin{array}{l}\text { Produktionskosten } \\
\text { (nucl.) } \\
\text { (konv.) }\end{array}$ & $\begin{array}{l}3,0 \mathrm{DM} / \mathrm{kg} \\
0,5 \mathrm{DM} / \mathrm{kg}\end{array}$ & $\begin{array}{l}0,116 \mathrm{DM} / \mathrm{kg} \\
0,12 \mathrm{DM} / \mathrm{kg}\end{array}$ \\
\hline
\end{tabular}

$30 \%$ einer Anlage von $222 \mathrm{MW}(\mathrm{e})$ entspricht. Infolge der mit steigender Anlagegróße sinkenden spezifischen Anlagekosten können wir hier mit etwas niedrigeren spezifischen Investitionskosten als im vorigen Fall rechnen, namlich mit etwa $1200 \mathrm{DM} / \mathrm{kW}$ (e). Es wird wieder vorausgesetzt, daß die Investitionskosten eines Chemiereaktors nicht wesentlich hoher sind als die eines normalen Leistungsreaktors. Der Reaktor wüde einschließlich Turbinen für die Erzeugung elektrischer Energie aus der Restenergle des Reaktors etwa 265 Mill. DM kosten. Wegen der starken Verunreinigung des chemischen Produktes wird noch eine Dekontaminierungsanlage benötigt, die mit $100 \mathrm{Mill}$. DM eingesetzt wird, so daß die Gesamtınvestitionskosten etwa 365 Mill. DM betragen. Die jährlichen Festkosten und Betriebskosten können wie im vorigen Falle mit zusammen $30 \%$ der Investitionskosten, also mit 110 Mill. DM angenommen werden. Durch Stromgutschrift aus dem Verkauf von 1,7 Mrd. kWh elektrischer Energie werden 52 Mill. DM gewonnen, so daß sich die Produktionskosten auf 58 Mill. DM je $500000 \mathrm{t}=0,116$ DM je kg Stickstoffdioxyd belaufen. Der Herstellungspreis in konventionellen Anlagen gleicher Größe wird mit 0,12 DM je $\mathrm{kg}$ Stickstoffdioxyd angegeben. Das chemonucleare Verfahren zur Stickstoff-Fixierung würde demnach wirtschaftlich arbeiten, obwohl auch 
hierbei nur ein geringer Teil $(2,5 \%)$ der Strahlungsenergie des Reaktors in chemische Energie umgewandelt wird. - Etwas weniger günstig sind die Vergleiche von Steinberg ${ }^{11}$ ), der für eine Reihe verschiedener Anlagen zu einem Verhältnis von 1 bis 2,9 der chemonuclearen und konventionellen Produktionskosten kommt. - Fixierter Stickstoff wird hauptsächlich als Düngemittel in Form von Ammoniumsulfat oder Ammoniumnitrat und nicht als Salpetersäure abgegeben. Auf dieser Basis sind die Kosten des nach konventionellen Methoden fixierten Stickstoffs sicher um den Faktor zwei niedriger als die des in Kernreaktoren fixierten Stickstoffs, selbst wenn das chemonucleare Verfahren so günstig ist, wie in der vorausgegangenen Diskussion angenommen wurde.

Die aufzuwendende Reaktionswärme für $1 / 2 \mathrm{~N}_{2}+$ $\mathrm{O}_{2} \longrightarrow \mathrm{NO}$, ist $8,1 \mathrm{kcal} / \mathrm{Mol}$, entsprechend $0,2 \mathrm{kWh} / \mathrm{kg}$ NO.. Im direkten Chemonuclear-Verfahren benötigen wir unter den gegebenen Bedingungen nahezu $12 \mathrm{kWh}$ Reaktorenergie je kg NO${ }_{2}$ (Tab. 4), d. h. der Wirkungsgrad ist etwa 2,0\%. Aber auch das konventionelle Verfahren, das über die Ammoniak-Synthese nach Haber-Bosch mit anschließender Verbrennung des Ammoniaks verläuft, erreicht nur einen energetischen Wirkungsgrad von $2 \%$. Noch schlechter schneidet der Birkeland-Eyde-ProzeB ab, bei dem die StickoxydSynthese aus Luft in einem Lichtbogen erzwungen wird. Der Wirkungsgrad ist hier nur $0,4 \%$.

\section{Ausblick}

Es ist natürlich einstweilen kein großer Anreiz, nach einem völlig neuartigen, bisher unerprobten Verfahren die Kernenergie in chemische Energie umzuwandeln und damit ein Produkt herzustellen, das mit konventionellen Mitteln billiger oder zumindest nicht teurer zu produzieren wäre.

Damit erhebt sich die Frage, durch welche Entwicklungen die Kosten eines im Chemie-Kernreaktor erzeugten Produktes gesenkt werden können. Es ist bekannt, daß die spezifischen Investitionskosten einer Anlage mit der Größe der Anlage abnehmen. Eine Vergrößerung der vorher diskutierten chemonuclearen Stickoxyd-Fabrik auf die zehnfache Jahreskapazität wäre durchaus möglich und würde eine stärkere Verringerung des Produktionspreises nach sich ziehen, als es bei einer entsprechenden Vergrößerung einer konventionellen Anlage zu erwarten wäre. Bei zu großen Anlagen tritt aber die Schwierigkeit des Absatzes und des Transports solch großer Mengen eines Produktes auf, und die Erniedrigung der Produktionskosten durfte durch die Erhöhung der Transportkosten kompensiert werden.

Da die Probleme des Chemie-Kernreaktors aufs engste mit den Problemen der Kernenergie-Erzeugung verbunden sind, werden die Fortschritte auf dem Gebiet der Kernenergie auch die wirtschaftlichen Grundlagen der Chemie-Kernreaktoren günstig beeinflussen. Von dieser Seite ist sicher eine Verbesserung zu erhoffen.

Schließlich sind auch günstige Momente aus der Grundlagenforschung der Strahlenchemie zu erwarten. Einige der bekannten strahlenchemischen Reaktionen werden in Gegenwart geringer Mengen eines Zusatzstoffes, der wie ein Katalysator wirkt, beschleunigt, verlangsamt oder in andere Richtungen gelenkt. Z. B. erhöht die Zugabe von 1\% Tetrachlorkohlenstoff die Glykol-Ausbeute bei der Bestrahlung von Methanol in der Gasphase auf das Doppelte ${ }^{12}$ ). Ein anderes Beispiel ist Kohlendioxyd, das gegen Strahleneinwirkung so resistent ist, daß es als Kühlmittel im Reaktorkreislauf eingesetzt werden kann. In Gegenwart von nur $1 \%$ Stickstoffmonoxyd oder Stickstoffdioxyd wird es jedoch mit einem G-Wert von 9 in Kohlenmonoxyd und Sauerstoff gespalten ${ }^{21}$ ). Durch Zusatzstoffe können demnach die Ausbeuten einiger chemonuclearer Reaktionen beträchtlich erhöht werden, und es ist zu hoffen, daß die weiteren Untersuchungen von strahlenchemischen Systemen Wege eröffnen, die die Wirtschaftlichkeit der diskutierten Verfahren verbessern.

Vielleicht werden auch neuartige Verbindungsklassen von technischem Interesse durch strahlenchemische Prozesse zugänglich: z. B. läßt sich unter gewissen BestrahJungsbedingungen aus Kohlenoxyd Kohlensuboxyd $(\mathrm{O}=\mathrm{C}=\mathrm{C}=\mathrm{C}=\mathrm{O})$ herstellen $\left.{ }^{21}\right)$, dessen Bedeutung für die chemische Industrie noch nicht abgeschätzt werden kann, da dieses Produkt in technischem Maßstab noch nirgends zur Verfügung steht. Als sehr energiereiche und reaktionsfreudige Verbindung würde sie sicher eine gute Zukunft haben.

Schließlich sei noch die strahlenchemische Reaktion zwischen Stickstoff und Kohlendioxyd erwähnt ${ }^{21}$ ), die eine Kombination der Stickstoff-Fixierung und Kohlenoxyd-Bildung ist:

$1 / 2 \mathrm{~N}_{2}+\mathrm{CO}_{2} \longrightarrow \mathrm{NO}+\mathrm{CO}$.

Bei einem G-Wert von annahernd 10 werden bei dieser Reaktion nahezu 33\% der im gasförmigen System absorbierten Strahlenenergie gespeichert, so daß man in diesem Falle wirklich von einer lohnenden Umwandlung von Kernenergie in chemische Energie sprechen konnte.

Beim gegenwärtigen Stand der Technik scheint der Einsatz von Chemie-Kernreaktoren noch nicht sehr verheißungsvoll. Auch wenn durch weitere Erfahrungen und neuere Erkenntnisse die eigentlichen Herstellungskosten einiger Produkte unter die konventionellen Herstellungskosten gesenkt werden könnten, so wird noch immer die radioaktive Verseuchung der chemischen Reaktionsprodukte die Anwendbarkeit der Chemie-Kernreaktoren hemmen. Die radioaktiven Spaltprodukte, die im Glasfaser- oder Staubphasenreaktor vom chemischen Reaktionsgut eingefangen werden, kann man wahrscheinlich weitgehend entfernen. Allerdings müssen die extrem hohen Dekontaminationsfaktoren, die bei der Aufarbeitung der Kernbrennelemente gefordert werden $\left(10^{7}\right.$ bis $\left.10^{8}\right)$, noch weit uberschritten werden, wenn man das Reaktionsprodukt nicht bis zum weitgehenden Zerfall der aktiven Spaltprodukte, d. h. bis zu 20 Jahren, lagern will. Aber noch schwieriger oder sogar unmöglich dürfte die Reinigung aller kohlenstoffhaltigen Verbindungen sein, die bei diesen die durch Neutronen-Einfang gebildeten aktiven ${ }^{14} \mathrm{C}$-Atome (Halbwertszeit 5600 Jahre) teilweise fest in die Molekeln eingebaut und damit nicht abtrennbar sind. Bei den sehr strengen Bestimmungen der Strahlenschutzverordnung in Deutschland dürfte damit der Vertrieb solcher Substanzen nahezu unmöglich sein.

Eingegangen am 29. August 1963 [B 1657] 


\section{Literatur}

1) Nucleonics 20, Nr. 12; 18 (1962).

2) F. Giesel, Verh. dtsch, physik. Ges, 2, 9 [1900], Ber. dtsch. chem. Ges. 35, 3608 [1902]; P. Curie u. A. Debjerne, C. R. hebd. Séances Acad. Sci. 132, 770 [1901]; H. Becquerel, C. R. hebd. Séances Acad. Sci. 133, 709 [1901]; W. Ramsay u. F. Soddy, Proc. Roy. Soc. [London], 72, 204 [1903].

3) S. z. B. Nachr, aus Chemie und Technik 3, 151 [1955].

4) S. z. B. Nachr. aus Chemie und Technik 6, 297 [1958].

5) A. O. Allen: The Radiation Chemistry, of Water and Aqueous Solutions, Princeton, N. J., 1961.

6) D. Schulle-Frohlinde u. K. Eiben, Z. Naturforsch. 17a, 445 [1962].

7) Radiation, a Tool for Industry, A. D. Little Corp., Cambridge, Mass., 1959.

8) H. Krauch, Kerntechnik 3, 1; 4 [1961].

9) S. Glasstone: Principles of Nuclear Reactor Engineering, Princeton, N. J , 1955.

10) H. Krauch, W. Baur, K. Jecht u. W. Kunz: Chemie-Kernreaktoren, Dechema-Monographie, Bd. 42, S. 145, Weinheim/Bergstr. 1962.

11) M. Steinberg, Advances in Nucl. Sci. Technology 1, 247 [1963].

12) A. K. Wiebe, W. P. Conner u. G. W. Kinzer, Nucleonics 19, Nr. 2, 50 [1961].

13) M. Steinberg u. L. Green, Brookhaven Nat. Lab., Bericht BNL 5755 (1960).

14) D. A. Landsman u. J. E. Butterfield, Atomic Energy Research Establ., Bericht AERE-R-3625 (1961).

15) N. A. Proskurnin u. Y. M. Kolotyrkin, Proc. 2nd. Intern. Conf. Peaceful Uses Atomic Energy, Geneva 1958, 29, 52 [1959]; E. V. Barelko, L. I. Kartashova, P. N. Komarow u N. A. Proskurnin, Large Radıation Sources in Industry, Intern. Atomic Energy Agency, Wien 1960, Bd. 1, S. 407
16) D. Cleaver, E. Collinson u. F. S. Daynton, Trans Faraday Soc. 56,1640 [1960].

17) C. P. Fritsch, J. Cusak, R. I. Miller u. M. R. Gustavson, Chem. Engng. Progr, 57, Nr. 3, 37 [1961].

18) Y. A. Sorokin u. S. Y Pshezhetski, Ber. Akad. Wiss. UdSSR 138, 880 [1961].

19) D. A. Landsman, Nuclear Engng. 7, Nr. 69, 50 [1962].

$\left.{ }^{20}\right)$ D. Harteck u. S. Dondes, Nucleonics 14, Nr. 7, 22 [1956].

21) D. Harleck u. S. Dondes, Z. Elektrochem., Ber. Bunsenges phys. Chem. 64, $983[1960]$; D. Harleck, S. Dondes u. D. E. Neil, Large Radiation Sources in Industry, Intern. Atomic Energy Agency, Wien 1960, Bd. 1, S. 231.

22) R. F. R. Coekelbergs u Mitarb., ebenda, Bd. 1, S. 191.

$\left.{ }^{23}\right)$ J. K. Dawson u. F Moseley, ebenda, Bd. 1, S. 223.

24) M. Steinberg, L. Green u. J. R. Powell, A. I. Ch. E. Journal 7,329 [1961].

25) Siehe auch Nucleonics 19, 2, 47 [1961].

29) P. Harteck u. S. Dondes, J. chem. Physics 23, 902 [1955]; 26, 1727 [1957]

27) J. G. Burr, Atomics International, Bericht NAA-SR 5350 VII-37 (1960)

28) E. Hayon u. J.J. Weiss, J chem Soc [London] 1961, 3970

29) L. E. Crean, L. S. Nims u J. J. Martun, Chem Engng Progr. 56, Nr. 10, 72 [1960].

3i) P. Harteck u. S. Dondes, Nucleonics 15, 8; 94 [1957].

31) G. Lellouche u. M. Steinberg, Brookhaven Nat. Lab, Bericht BNL 574 (1959).

32) Aerojet-General Nucleonics, Bericht AGN 3048 (1959)

33) M. Steinberg, J. R. Powell u. L Green, Brookhaven Nat. Lab., Bericht BNL 602 (1962).

34) J. E. Ullmann, Advances in Nucl. Sc1. Technology 1, 225 [1962]. 